\title{
Age Invariant Face Recognition using K-PCA and K-NN on Indian Face Age Database (IFAD)
}

\author{
Reecha Sharma \\ Department of Electronics and Communication \\ Engineering, \\ Punjabi University, \\ Patiala-147002, Punjab, India
}

\author{
M.S. Patterh \\ Department of Electronics and Communication \\ Engineering, \\ Punjabi University, \\ Patiala-147002, Punjab, India
}

\begin{abstract}
In this paper a comparative analysis of K-Principle Component Analysis (K-PCA) and K-Nearest Neighbor (K$\mathrm{NN}$ ) classifier is done for age invariant face recognition using Indian Face Age Database (IFAD). IFAD is a real time and wild in face database which can be used for face recognition at different variation parameters. These variations can be pose, illumination, occulation, and age. In this paper age variation is prime issue for face recognition. The IFAD database consists of 55 subjects. The images are not preprocessed. In IFAD face detection is done by Viola Jones face detection algorithm. It is analyze that K-NN gives high classification rate but take more execution time at high values of $\mathrm{K}$ components. On the other hand Euclidean distance gives less classification rate and less execution time at high values of $\mathrm{K}$ components. So K-NN can perform better for age invariant face recognition if its execution time improved in future.
\end{abstract}

\section{Keywords}

Image Processing, Feature Extraction, Face Recognition and Machine Vision.

\section{INTRODUCTION}

Face recognition can be used for finding identity of a person, human computer interface (HCI) or for security purposes. Face has complex, multidimensional and meaning full image. Face recognition by human computer interface is a challenging task because overall configuration of face is similar. There are three basic steps for face recognition.

- $\quad$ Face image preprocessing

- Feature extraction

- Face image classification

Face image preprocessing: Due to the limited dynamic ranges of current imaging and display devices, images captured in real world scenes with high dynamic ranges usually exhibit poor visibility of either over exposed or shadows and low contrast, to cope with this problem, various image processing techniques have been developed. Some of those techniques are spatially independent methods, like gamma adjustment, logarithmic compression, histogram equalization (HE), and levels/curve methods.

Feature extraction: Feature extraction, a special form of dimensionality reduction, is the process of computing a compact numerical representation that can be used to characterize a pattern. The design of descriptive feature for a specific application is the main challenge in building pattern recognition systems.
Classification: Basic-level categorization is the most common entry point for classification but the basic level of classification is not enough information to identify faces. This occurs if information outside the long-term memory matches the information to be retrieved; the retrieval process helps in face recognition.

In this proposed methodology face detection is done by Viola Jones (VJ). Feature extraction can be local feature based (mouth, eyes, nose, etc.) or global feature based (full face image). In proposed methodology feature extraction is done by PCA. Face image classification is done by Euclidean distance (ED) or by Nearest Neighbor (NN) classifier.

IFAD database is used testing recognition rate. IFAD is a wild face database which has real time images without any preprocessing. IFAD provides large variation of age characteristic of a subject from childhood to old age. There is a series of phases of variation in appearance of face of an individual. Every subject shows variation in hairstyle, weight, skin texture etc. The IFAD also shows variation in illumination, pose, occulation, expression.

The rest of the paper is organized as follows: in section 2 reviews of PCA based existing techniques of face recognition are discussed. In Section 3 PCA algorithm is explained. Section 4 elaborates the proposed methodology for age invariant face recognition. In section 5 experimental results are discussed for proposed methodology for age invariant face recognition. Section 6 concludes the proposed methodology.

\section{REVIEW OF PCA BASED EXISTING TECHNIQUES OF FACE RECOGNITION}

PCA is a dominant technique for extracting features from high-dimensional data sets, which corresponds to extracting the eigenvectors that are associated with the largest eigenvalues from the input distribution. This eigenvector analysis has already been widely used in face processing. Sirovich-Kirby (1987) and Kirby-Sirovich (1990) explained that PCA can be used efficiently to describe geometry of the faces [1] [2]. They explain that from few Eigen vectors, faces can be reconstructed for representation. Turk and Pentland (1991) made use of Eigenfaces for face detection [3]. A kernel principal component analysis (KPCA) is a nonlinear extension of a PCA. In KPCA nonlinear mapping used to map the input space into a feature space. After that principal components are calculated in that feature space [4]. In 2DPCA $2 \mathrm{D}$ image matrices is calculated instead of $1 \mathrm{D}$ vector. It is the advantage of 2DPCA that there is no need to transform image matrix into a vector prior to feature extraction [5]. When diagonal PCA is combined with PCA it gives more accuracy 
as compare to PCA, 2DPCA or DiaPCA. When PCA is combined with some other extended form of PCA it gives higher recognition rate as compare to simple PCA [6]. In modular PCA image is divided into parts and weight vector are calculated for each part. These weights are more representative for local information of face. The modular PCA gives better results for variation in expression and illumination [7]. SubXPCA eliminates the problem of PCA by extracting principal components with global and local information [8]. A neuro fuzzy approach which is a combination of PCA and ANFIS perform much better than PCA or LDA. PCA+ANFIS also gives better results in terms of accuracy, sensitivity and specificity than feed forward neural network (FFBNN) approach [9].

\section{PCA}

PCA is one of the most successful techniques that have been used in image recognition and compression. PCA is a statistical method under the broad title of factor analysis. PCA is a classical and the benchmark algorithm for face recognition [10]. The jobs, which PCA can do, are prediction, redundancy removal, feature extraction, data compression, etc. Because PCA is a classical technique, which can do something in the linear domain, applications having linear models are suitable, such as signal processing, image processing, system and control theory, communications, etc. The main idea of using PCA for face recognition is to express the large 1-D vector of pixels constructed from 2-D facial image into the compact principle components of the feature space. This can be called Eigen space projection. Eigen space is calculated by identifying the eigenvectors of the covariance matrix derived from a set of facial images (vectors) [11].The advantage of PCA comes from its generalization ability. It reduces the feature space dimension by considering the variance of the input data. The method determines which projections are preferable for representing the structure of the input data. Those projections are selected in such a way that the maximum amount of information (i.e. Maximum variance) is obtained in the smallest number of dimensions of feature space [12].

In proposed methodology training set of $X n$ images of size $32 \times 32$ are represented by vectors of size $X^{2}$. Each face is represented by $X^{l} ; X^{2} ; X^{3} ; \ldots \ldots \ldots ; X^{M} . \mathrm{NxN}$ matrix stores the feature vector. Now, this two dimensional vector is changed to one dimensional vector. Fig. 1 . shows the flow chart of PCA algorithm.

Steps of PCA algorithm used in proposed methodology are explained below:

Step 1: Obtain a set of pixel from an image.

$$
f_{d}^{\prime}=x_{1}, x_{2} \ldots x_{N}
$$

Where $x_{1}, x_{2} \ldots \ldots x_{N}$ are the pixel values of an image. $\mathrm{N}$ is the total number of pixels in an image,

Step 2: Compute the Mean value.

$$
\mu=(1 / N) \sum_{i=1}^{N} x_{i}
$$

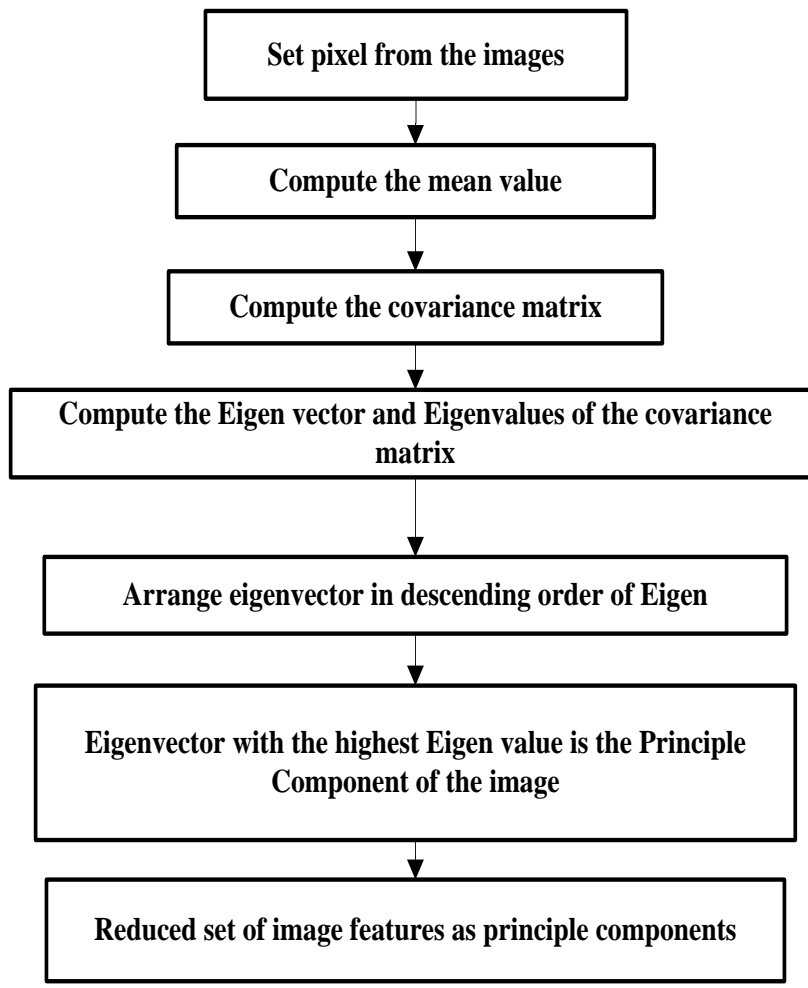

Fig.1. Flow chart of PCA

Step 3: Compute the covariance matrix.

$$
K=(1 / N) \sum_{i=1}^{N}\left(x_{i}-\mu\right)\left(x_{i}-\mu\right)^{T}
$$

Step 4: Compute the Eigen vectors $e$ and Eigen values $\lambda$ of the covariance matrix $K$.

If $K$ is a square matrix, a non-zero vector $e$ is an eigenvector of $K$ if there is a scalar $\lambda$ (Eigen value) such that

Solve $K e=\lambda e$

Step 5: Arrange eigenvectors in descending order of Eigen values.

$\lambda_{1} \geq \lambda_{2} \geq \ldots \ldots . \lambda_{n}$

The eigenvector with the highest Eigen value is the principle component of the image.

Step 6: PCA reduced the dimension by keeping direction $e$ such that $\lambda<T$

Step 7: Make the reduced set of image features as score values.

\section{PROPOSED METHODOLOGY FOR FACE RECOGNITION}

In the proposed methodology IFAD database is used for comparative analysis of KPCA and NN classifier for face recognition. There are 55 subjects (persons) in the IFAD. Each subject has different number of images. There are in total 3296 images in IFAD. The IFAD is available with $32 \times 32,64 \times 64,96 \times 96$ and $128 \times 128$ dimensions. In $32 \times 32$ IFAD, the total number of images are 3294 out of 3296 
images, 2 were rejected by the algorithm In IFAD 32x32 dimension images has 1414 number of are male images and 1882 are female images. Fig.2 shows the flow chart of proposed methodology. Here IFAD data set is divided into two parts: training and test set. The images in training set are different from testing set. The ratio of training and testing set is $7: 3$. From total images of IFAD, $70 \%$ images are training and $30 \%$ images are testing. Table 1 shows the details of IFAD images used in proposed methodology.

Table 1. Details of IFAD images used in proposed methodology

\begin{tabular}{|l|l|}
\hline Number of subjects & 55 \\
\hline Total number of images & 3296 \\
\hline Number of Male images & 1414 \\
\hline Number of female images & 1882 \\
\hline Number of training images & 2307 \\
\hline Number of testing images & 989 \\
\hline
\end{tabular}

After preparing IFAD datasets for training and testing, $K$ principle components are extracted by using PCA algorithm. K-PCA was chosen because of its superiority in dimensional reduction ability. In K-PCA $K$ components with most variant are selected. As most of information of a face is carried in components with highest variance.

$$
C=\frac{1}{M} \sum_{n=1}^{M} \Phi_{n} \Phi_{n}^{T}=A A^{T}
$$

K-PCA chosen by $\mathrm{K}$ feature subspace selector are given to classifiers. Here in proposed methodology two classifiers Euclidian distance and Nearest Neighbor are used.

The Euclidian distance

$\varepsilon_{k}^{2}=\left\|\left(\Omega-\Omega_{k}\right)^{2}\right\|$

where $\Omega_{k}$ is a vector describing the $k$ th face class. The face classes $\Omega_{k}$ are considered by averaging the consequences of the Eigen Face representation over a small number of face images (as few as one) of each being. A face is confidential as "unknown", and optionally used to produce a new face class.

In face recognition, the Nearest Neighbors $(\mathrm{NN})$ is a nonparametric method used for classification and regression. In both cases, the input consists of the $\mathrm{k}$ closest training samples in the feature space. It is also called k-NN. The output depends on whether k-NN is used for classification or regression. In k-NN classification, the output is a class membership. An object is classified by a majority vote of its neighbors, with the object being assigned to the class most common among its knearest neighbors ( $\mathrm{k}$ is a positive integer, typically small). If $\mathrm{k}=1$, then the object is simply assigned to the class of that single nearest neighbor. In k-NN regression, the output is the property value for the object. This value is the average of the values of its $\mathrm{k}$ nearest neighbors.
The test set images follow the same steps as training set images shown in flow chart of fig.2. Classifier evaluator compares the test image with training set images and best match gives the classification rate.

IFAD Dataset

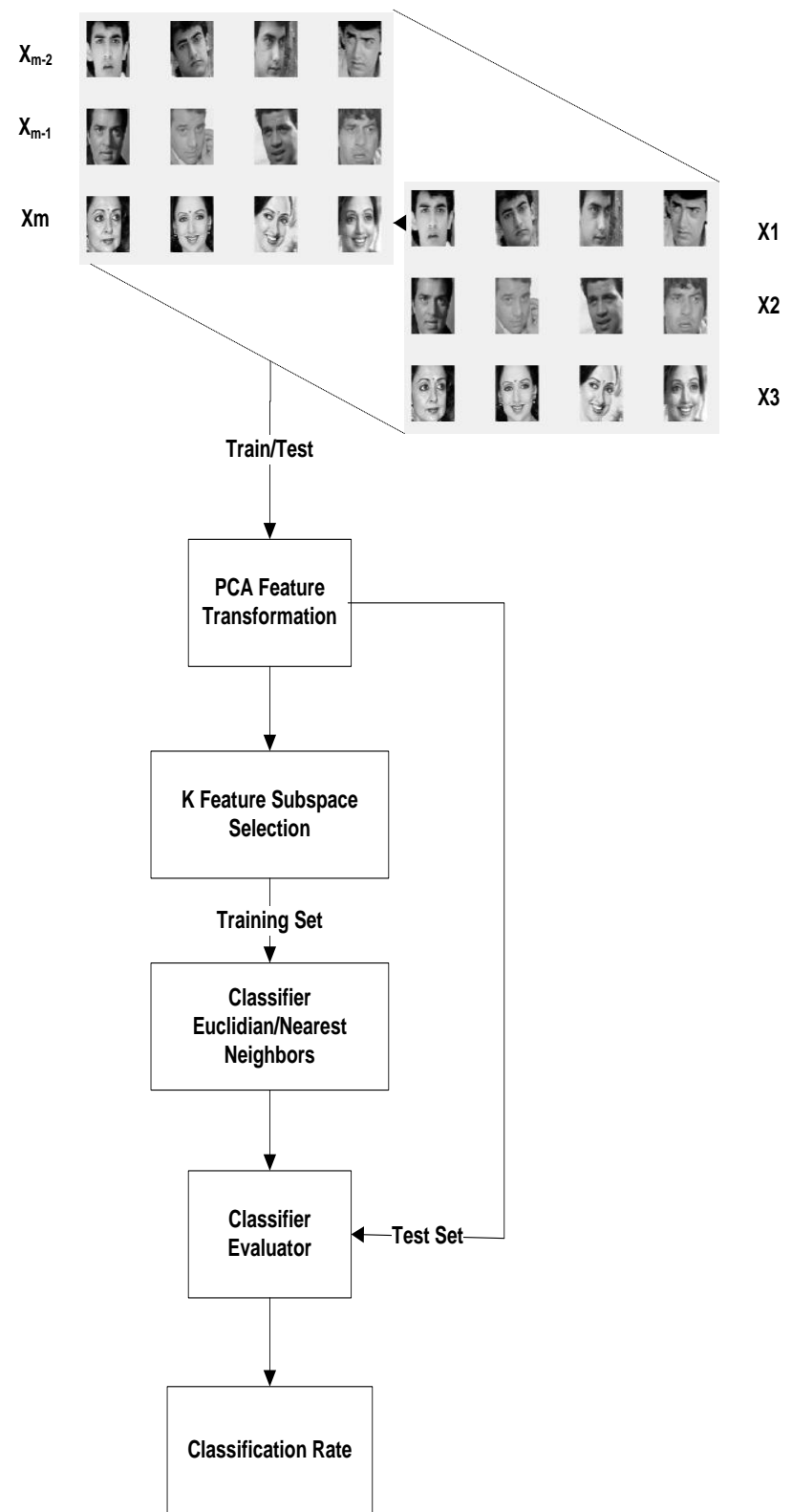

Fig.2.Flow chart of proposed methodology for face recognition using IFAD

\section{EXPERIMENTAL RESULTS AND DISCUSSIONS}

In proposed methodology for age invariant face recognition the selection of $\mathrm{K}$ component is done very carefully. At low value of $\mathrm{K}$, the classification rate is very low. But as $\mathrm{K}$ increases the classification rate improves as shown in table 2 .

Table 2. Classification rate of K-PCA and K-NN at different values of $K$ components

\begin{tabular}{|c|c|c|}
\hline Components & \multicolumn{2}{|c|}{ Classification rate (\%) } \\
\hline K & $\begin{array}{c}\text { K-PCA } \\
\text { (Euclidean }\end{array}$ & K-NN \\
\hline
\end{tabular}




\begin{tabular}{|c|c|c|}
\hline & Distance) & \\
\hline 10 & 0.066 & 0.066 \\
\hline 20 & 0.089 & 0.089 \\
\hline 30 & 0.1123 & 0.1123 \\
\hline 50 & 0.2144 & 0.2144 \\
\hline 100 & 0.24 & 0.2625 \\
\hline 200 & 0.4667 & 0.6875 \\
\hline 300 & 0.4417 & 0.7333 \\
\hline
\end{tabular}

As seen from the table, the classification rate of K-PCA and $\mathrm{K}-\mathrm{NN}$ is same for $\mathrm{K}=10,20,30,50$. But after $\mathrm{K}=100$, there is variation in classification rate of both classifiers. At $\mathrm{k}=200 \mathrm{~K}$ PCA gives $0.4667 \%$ classification rate and $\mathrm{K}-\mathrm{NN}$ gives $0.6875 \%$ classification rate which is much higher than $\mathrm{K}$ PCA. When value of $\mathrm{K}$ is chosen as 300 , classification rate of $\mathrm{K}-\mathrm{PCA}$ fall down but increases in K-NN.

For $\mathrm{K}=100$ the maximum recognition achieved by Euclidian Distance was $24 \%$ and for K-NN it is $26.25 \%$ as shown in fig.3. The mean classification rate for Euclidian Distance is $19.49 \%$ and standard deviation is $3.34 \%$. The mean classification rate for $\mathrm{K}-\mathrm{NN}$ is $21.95 \%$ and standard deviation is $3.67 \%$. As seen at $\mathrm{K}=100$ the recognition rate is only $26.25 \%$ by $\mathrm{K}-\mathrm{NN}$ is very low. From this value it can be concluded that for high classification rate $\mathrm{K}$ can be improved.

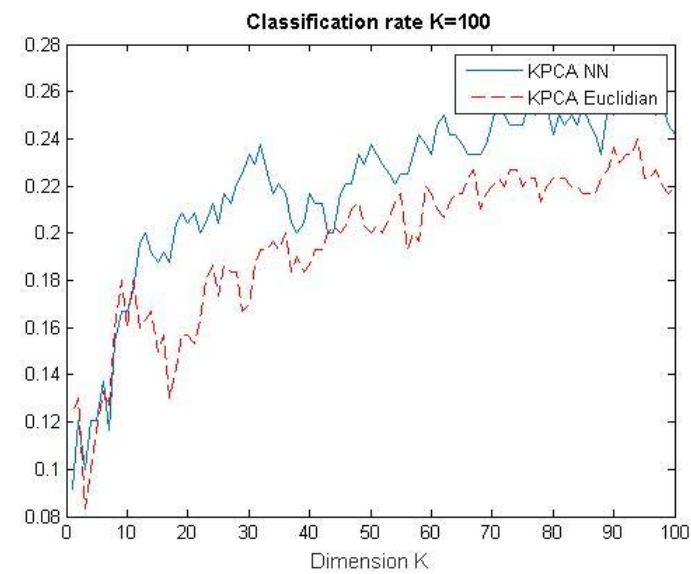

Fig.3 Classification rate at $\mathrm{K}=100$

In Fig. 4 for $\mathrm{K}=200$ the maximum recognition achieved by Euclidian Distance was $46.67 \%$ and for $\mathrm{K}-\mathrm{NN}$ it is $68.75 \%$. The mean classification rate for Euclidian Distance is $40.64 \%$ and standard deviation is 3.6. The mean classification rate for $\mathrm{K}-\mathrm{NN}$ is $62.11 \%$ and standard deviation is $2.1 \%$. As seen at $\mathrm{K}=200$ the recognition rate is only $62.11 \%$ by $\mathrm{NN}$ is very low. It can noted from the fig. 4 that K-NN based method has low standard deviation and tends to give exact results without so much error.

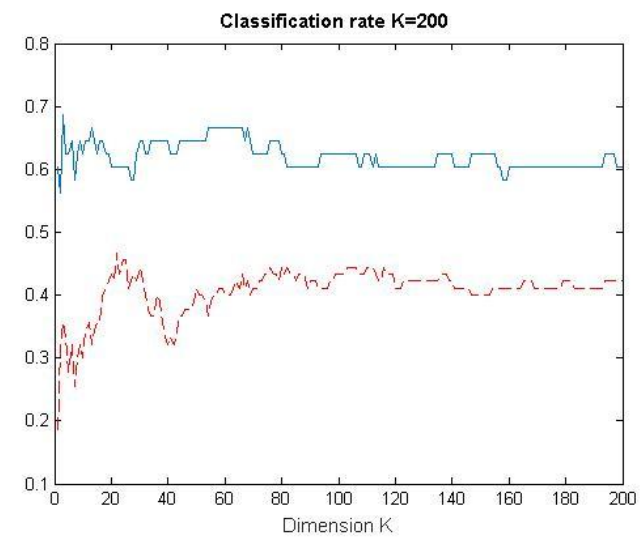

Fig.4 Classification rate at $\mathrm{K}=\mathbf{2 0 0}$

Fig.7. For $\mathrm{K}=300$ the maximum recognition achieved by Euclidian Distance was $44.17 \%$ and for K-NN it is $73.33 \%$ The mean classification rate for Euclidian Distance is $40.43 \%$ and standard deviation is $3.3 \%$. The mean classification rate for $\mathrm{K}-\mathrm{NN}$ is $72.09 \%$ and standard deviation is $1.54 \%$ As seen at $\mathrm{K}=300$ the recognition rate is only $73.33 \%$ by $\mathrm{K}-\mathrm{NN}$ is very low. From this value it can be concluded that for high classification rate $\mathrm{K}$ can be improved.

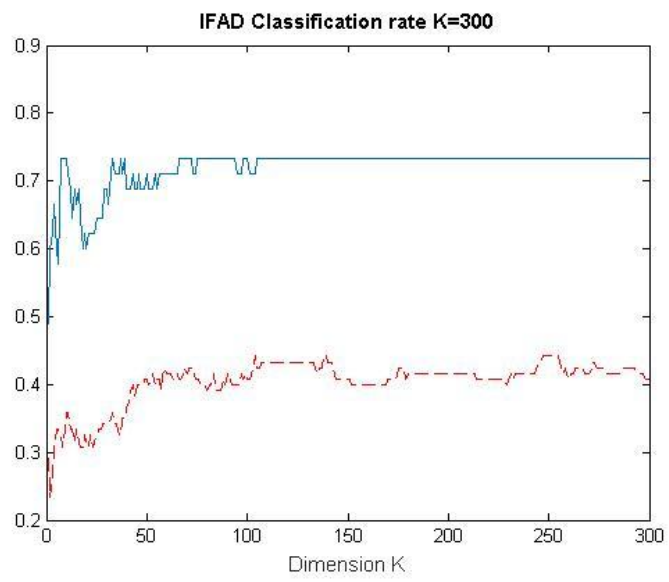

Fig.5 Classification rate at $\mathrm{K}=300$

Fig.7 shows the execution time K-PCA and K-NN at different values of $\mathrm{K}$. It is seen that as value of $\mathrm{K}$ components increases the execution time increases rapidly. Execution time of $\mathrm{K}$ PCA is less as compare to K-NN. K-NN classifier gives more accuracy as compare to K-PCA but it is slower.

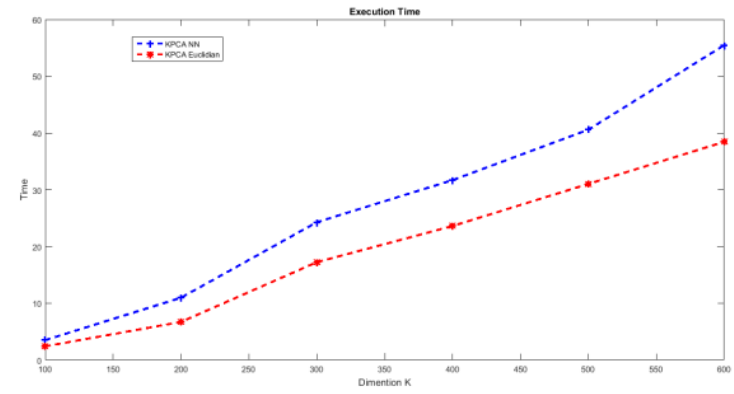

Fig.6 Execution time at different values of $\mathrm{K}$

Fig.7 shows the Error Rate for IFAD using K-PCA with K$\mathrm{NN}$ at different values of $\mathrm{K}$. Error Rate stabilizes at around $\mathrm{k}=230$ and minimum error at $\mathrm{K}=211$. 


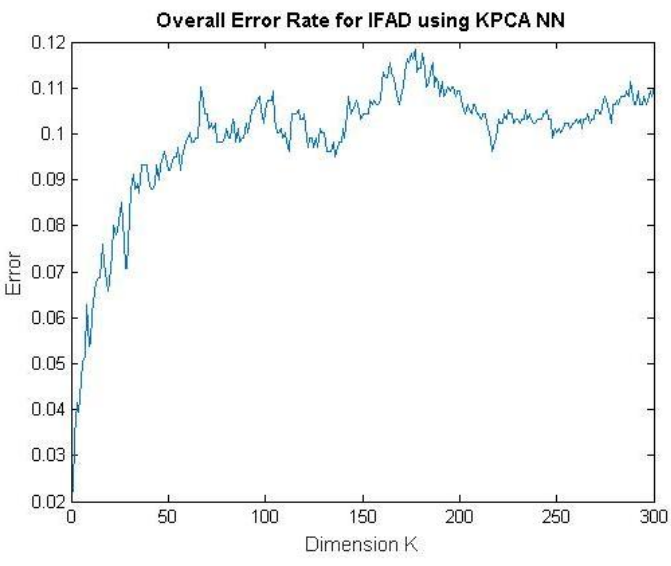

Fig.7 Overall error rate for IFAD using KPCA and NN

\section{CONCLUSION AND FUTURE SCOPE}

This paper presents the comparative study of K-PCA and K$\mathrm{NN}$ for age invariant face recognition on IFDA data face. It is seen that k-principle components are chosen very carefully. At $\mathrm{k}=100$ it gives both classifiers gives less classification rate but as value of $\mathrm{k}$ increased up to 300 , it give high classification rate. Euclidean distance gives $44.17 \%$ and $\mathrm{k}-\mathrm{NN}$ gives $73.33 \%$ which is quite good for IFAD database. But execution time k-NN is high. So it is concluded that K-NN out perform as compare to Euclidean distance. In future K-PCA and k-NN can be improved for fast response. Age estimation based on these classifiers can be done in future work.

\section{ACKNOWLEDGMENTS}

I would like to express my greatest gratitude to my guide Dr. M.S Patterh Department of ECE, Punjabi University for his continuous support for the paper from initial advice \& contacts in the early stages of conceptual inception \& through ongoing advice $\&$ encouragement to this day. I would like to thank the anonymous reviewers for their constructive comments.

\section{REFERENCES}

[1] M. Kirby and L. Sirovich, "Application of the KarhunenLokve Procedure for the Characterization of Human Face," IEEE Transactions on Pattern Analysis and Machine Intelligence, vol. 12, no. 1, January 1990.

[2] L. Sirovitch and M. Kirby, "Low-Dimensional Procedure for the Characterization of Human Faces," Journal of the Optical Society of America A, vol. 2, pp. 519-524, March 1987.

[3] Turk Matthew and Alex Pentland, "Eigenface for Recognition," Journal of Cognitive Neuroscience, vol. 3, no. 1, pp. 71-86, 1991.

[4] Kim Kwang In, Jung Keechul, and Kim Hang Joon, "Face recognition using kernel principal component analysis," Signal Processing Letters, IEEE, vol. 9, no. 2, pp. 40-42, 2002.

[5] Jian Yang, David Zhang, Alejandro F. Frangi, and Jingyu Yang, "Two-Dimensional PCA:A New Approach to Appearance-Based Face Representation and Recognition," IEEE TRANSACTIONS ON PATTERN
ANALYSIS AND MACHINE INTELLIGENCE, vol. 26, no. 1, pp. 131-137, Jan 2004.

[6] Reecha Sharma and M.S Patterh, "A Systematic Review of PCA and Its Different Form for Face Recognition," International Journal of Scientific \& Engineering Research, vol. 5, no. 7, pp. 1306-1309, July 2014.

[7] Rajkiran Gottumukkal and Vijayan K.Asari, "An improved face recognition technique based on modular PCA approach," Pattern Recognition Letters 25 (2004) 429-436, vol. 25, pp. 429-436, 2004.

[8] [8] Kadappa Vijayakuma and Negi Atul, "Computational and space complexity analysis of SubXPCA," Pattern Recognition, vol. 46, no. 8, pp. 2169-2174, 2013.

[9] Reecha Sharma and M.S Patterh, "Pose Invariant Face Recognition using Neuro-Fuzzy Approach," IOSR Journal of Computer Engineering (IOSR-JCE), vol. 17, no. 3 , pp. $20-27,2015$.

[10] Gundimada Satyanadh and Asari Vijayan, "Face alignment and adaptive weight assignment for robust face recognition," Advances in Visual Computing, pp. 191-198, 2005.

[11] Zhao Wenyi, Chellappa Rama, P Jonathon Phillips, and Rosenfeld Azriel, "Face recognition: A literature survey," ACM computing surveys (CSUR), vol. 35, no. 4, pp. 399-458, 2003.

[12] Sattar Abdul, Aidarous Yasser, Le Gallou Sylvain, and Seguier Renaud, "Face alignment by $2.5 \mathrm{~d}$ active appearance model optimized by simplex," in International Conference on Computer Vision Systems (ICVS), 2007, pp. 1--10.

\section{AUTHOR'S PROFILE}

Reecha Sharma is doing Ph.d in the field of Image Processing. She did her B.Tech. in Electronics and Instrumentation Engineering from MMEC Mullana, Ambala, India in 2003. She did M.E in Electronics, Instrumentation and Control Engineering from Thapar University Patiala, India in 2005. She has nine years of teaching experience. She has guided fifteen M.Tech. students. She has published over 30 research papers in International/National Journals and Conferences. Her area of research is Image Processing. At present she is working as Assistant Professor in Department of ECE, Punjabi University Patiala, Punjab, India.

M. S. Patterh, did his graduation in Electronics Engineering in 1988 from MITS Gwalior India, Masters from BITS Pilani India in 1993 and $\mathrm{Ph} \mathrm{D}$ in Electronics and Communication from PTU India. He is having over 23 years of teaching and over 13 years of research experience. At present he is working as Professor in Department of ECE, Punjabi University Patiala, India. His areas of interest include Signal Processing and Communication Engineering. He has published over 100 research papers in International/National Journals and Conferences. He is awarded twice Sir Thomas Memorial Ward prize by Institution of Engineers (India) for the research contributions in the area of Digital Communications. He has supervised six PhDs to date. He is member of IEEE, IETE, IE(I) and LSTE. He has completed projects worth $\$ 100,000$. 